Research Article

Animal Genetics

\title{
Determining minimum numbers of di-allelic diagnostic markers required to identify introgressions in diploid cross-species hybrid individuals from different types of inter- and backcross populations
}

\author{
Joseane Padilha da Silva ${ }^{1}$ (D) and Alexandre Rodrigues Caetano ${ }^{1}$ iD \\ ${ }^{1}$ Embrapa Recursos Genéticos e Biotecnologia, Parque Estação Biológica, Brasília, DF, Brazil
}

\begin{abstract}
Cross-species hybridizations have been extensively used to generate animals and plants better suited for draft and food and fiber production since Roman times, and are still important in current agricultural practices with growing uses especially in aquaculture. Diagnostic tools based on marker panels with sufficient numbers of markers for accurate identification of cross-species hybrid individuals from intercrossed and backcrossed populations are increasingly necessary for practical, accurate species-purity certification and management of commercial broodstocks. Minimal numbers of di-allelic markers with species-specific alleles required to accurately identify hybrid individuals in intercrossed and advanced backcrossed populations were estimated using power analysis, and ranged from 5 to $191(\alpha=.05)$, and from 7 to $293(\alpha=.01)$, considering backcross 1 (BC1) to BC6 populations, respectively. Numbers of markers required for accurate hybrid identification observed in simulated BC1 to BC6 populations ranged from 5 to 1,131 and 7 to 8,065 , considering error rates $\leq 5 \%$ and $\leq 1 \%$, respectively. Estimated and observed numbers of diagnostic markers required for accurate hybrid identification up to four generations of backcrossing fall within practical operational limits of most commercial platforms currently available for genotyping low-density SNP marker panels. Therefore, cost-effective assay panels could be developed to provide practical tools for accurate species-purity certification.
\end{abstract}

Keywords: Power test, hybrid identification, cross-species hybridization, broodstock management.

Received: October 01, 2019; Accepted: June 28, 2020.

\section{Introduction}

Artificial production of cross-species hybrids has been extensively used to generate animals and plants better suited for a diversity of uses such as draft and production of food and fiber since ancient Roman times (Adams et al., 2007), and still plays an important role in current agricultural practices, with growing use especially in aquaculture. Crossspecies hybrids of cultured fish are widely used (reviewed by Bartley et al., 2001) and account for a significant share of current finfish production. F1 hybrids are expected to perform better in captive production systems in respect to productivity and quality traits, as a consequence of the resulting positive heterosis, in spite of the lack of solid studies in many instances to accurately contrast production performances of hybrid populations with parental species (reviewed by Hashimoto et al., 2012).

Several fish hybrids produced in captivity have been shown to be fertile and can naturally generate progeny in both intercrosses with other hybrids and backcrosses with

Send correspondence to Alexandre Rodrigues Caetano. Embrapa Recursos Genéticos e Biotecnologia, Parque Estação Biológica, Avenida W5 Norte (Final), 70770-917, Brasília, DF, Brazsil. E-mail: alexandre.caetano@embrapa.br. parental species (Bartley et al., 2001), posing a threat to wild populations which co-inhabit river basins where hybrid aquaculture escapees can freely mate with parental species naturally isolated by non-genetic barriers (Hashimoto et al., 2012). Moreover, considering the lack of morphological differences between post-F1 hybrids and parental species reported in many instances (Hashimoto et al., 2011), one of the main challenges found in the large-scale use of hybridization is the potential contamination of pure parental broodstocks (Mair, 2007). Once unknown introgressions have occurred in captive broodstocks, expected results from genetic improvement programs established to breed fish with better productivity and quality traits may become unknowingly compromised, especially because observed high fecundity rates can act to rapidly disseminate introgressed germplasm.

Methods developed to infer population structure and assign individuals to populations using multilocus genotype data (Pritchard et al., 2000) are well established and have been used to assign individuals to populations, identify migrants and admixed individuals, in natural and captive populations, but may not be best suited for practical identification of advanced hybrids in routine testing. Molecular tools based on species-specific diagnostic alleles assayed by PCR-RFLP have been developed for identifying neotropical 
fish hybrids used for aquaculture (Hashimoto et al., 2010, 2011, 2012; Prado et al., 2011). These tools have been based on low numbers of unlinked markers (up to three) and have been shown to be fully effective in identifying F1 hybrids. However, adequate statistical frameworks to determine numbers of markers required to reliably identify hybrids in advanced intercrossed and backcrossed populations have not been developed and are essential for establishing optimal parameters for designing low-cost diagnostic marker panels based on proper numbers of markers for accurate speciespurity certification of individuals and management of commercial broodstocks, and monitoring of wild populations.

Current molecular tools for parentage verification, genetic-disease diagnostics, and genetic improvement of species used for food production are mostly based on SNP markers, as recent technologies have allowed for development of assay platforms that can genotype up to hundreds of thousands of markers in parallel, in highly automated processes which result in low costs and low error rates (Vignal et al., 2002). Basic statistical groundwork and formulae have been stablished to determine minimal numbers of unlinked di-allelic SNP markers with adequate inference power required for parentage verification (Baruch and Weller, 2008) and product tracking (Heaton et al., 2005) in farm animals, using approaches that allow decision making based on power analysis with minimal probabilities of errors. Work presented herein was performed to establish a basic statistical framework to compute minimal numbers of SNP markers with diagnostic (species-specific) alleles required to accurately identify, in routine testing, cross-species hybrid individuals from different types of inter- and backcrossed populations of diploid organisms, based on power analysis, and to verify these in simulated populations.

\section{Material and Methods}

\section{Determining minimal numbers of markers required for hybrid detection based on power analysis}

The established statistical framework is based on the use of bi-allelic SNP markers with species-specific alleles. Therefore, background work is required to identify cross- species SNPs, verified to be fixed for alternative alleles across large numbers of individual samples across different populations of a target species and one or more non-target species used for production of hybrids. Therefore, a target species needs to be shown to carry only a particular allele $(f(1)=1, f(2)=0)$, while other species used for hybridization have to be shown to carry only the alternative allele $(\mathrm{f}(1)=0$, $\mathrm{f}(2)=1$ ), at any useful SNP marker. Consequently, an F1 cross-species hybrids with the target species is expected to be heterozygous at all diagnostic SNPs $(\mathrm{F}(12)=1$; $f(1)=f(2)=0.5)$. A backcross of an F1 with the target species (BC1) results in allelic and genotypic frequencies as follows: $\mathrm{f}(1)=0.75, \mathrm{f}(2)=0.25 ; \mathrm{F}(11)=0.5, \mathrm{~F}(12)=0.5$, respectively. Table 1 shows expected allelic and genotypic frequencies for different types of inter (F1 and F2) and backcrossed (BC1 to BC6) hybrid populations from distinct, diploid, allogamous species, capable of generating fully fertile hybrid progeny, assuming random mattings and zero mutation, migration, selection and drift at every generation. For a given backcross level, the expected probability of observing the alternative allele at any diagnostic locus is $p_{0}$ (or $p$ under $H_{0}$, Table 1 ).

Considering $m$ independent loci, the number of occurrences of alternative alleles at diagnostic positions in the genome of an individual of a given backcross level follows a binomial distribution $X \sim B\left(m, p_{0}\right)$. A true hybrid is not identified if $\mathrm{X}=0$, therefore it is necessary to keep $P(X=0)$ below a certain level $\beta$ for accurate identification of true hybrids, that is:

$$
\begin{aligned}
& P(x=0)<\beta \\
& =\left(\begin{array}{c}
m \\
0
\end{array}\right) p_{0}^{0}\left(1-p_{0}\right)^{m}<\beta \\
& =\left(1-p_{0}\right)^{m}<\beta,
\end{aligned}
$$

as a result,

$$
m>\frac{\ln (\beta)}{\ln \left(1-p_{0}\right)}
$$

represents the minimal number of $m$ markers required for accurate identification of true hybrids, which can be determined by calculating the probability of observing an allele

Table 1 - Expected allelic and genotypic frequencies in different intercross and backcross populations between a target and a non-target species and respective hypothesis for statistical tests for hybrid identification.

\begin{tabular}{lccc}
\hline Population & Expected Allelic Frequencies $\mathrm{f}(1): \mathrm{f}(2)$ & Expected Genotypic Frequencies $\mathrm{F}(11): \mathrm{F}(12): \mathrm{F}(22)$ & Hypothesis \\
\hline Target Species & $1.0: 0.0$ & $1: 0: 0$ & - \\
F1 & $0.5: 0.5$ & $0: 1: 0$ & - \\
F2 & $0.5: 0.5$ & $0.25: 0.5: 0.25$ & $\mathrm{H}_{0}: p=0.75 ; \mathrm{H}_{1}: p<0.75$ \\
BC1 & $0.75: 0.25$ & $0.5: 0.5: 0$ & $\mathrm{H}_{0}: p=0.5 ; \mathrm{H}_{1}: p<0.5$ \\
BC2 & $0.875: 0.125$ & $0.75: 0.25: 0$ & $\mathrm{H}_{0}: p=0.25 ; \mathrm{H}_{1}: p$ \\
BC3 & $0.9375: 0.0625$ & $0.875: 0.125: 0$ & $\mathrm{H}_{0}: p=0.125 ; \mathrm{H}_{1}: p<0.125$ \\
BC4 & $0.96875: 0.03125$ & $0.9375: 0.0625: 0$ & $\mathrm{H}_{0}: p=0.0625 ; \mathrm{H}_{1}: p<0.0625$ \\
BC5 & $0.984375: 0.015625$ & $0.96875: 0.03125: 0$ & $\mathrm{H}_{0}: p=0.03125 ; \mathrm{H}_{1}: p<0.03125$ \\
BC6 & $0.9921875: 0.0078125$ & $0.984375: 0.015625: 0$ & $\mathrm{H}_{0}: p=0.015625 ; \mathrm{H}_{1}: p<0.015625$ \\
\hline
\end{tabular}


from a non-target species after $\mathrm{n}$ generations of backcrossing with a target species, as $p$ approaches zero. The correct identification of hybrid individuals occurs when a non-target allele is observed at any diagnostic SNP.

\section{Simulations}

Expected numbers of bi-allelic markers required to identify hybrids in all different intercross and backcross populations between a target and a non-target species considered were compared with observed numbers of markers based on simulated populations. R/qtl (Broman et al., 2003) was used to simulate SNP marker positions and genotypes for each evaluated population type based on a genetic map (Nunes et al., 2017) available for Tambaqui (Colossoma macropomum), a neotropical fish species with captive production in rapid expansion, commonly hybridized with closely related species (Hashimoto et al., 2011). A total of 7,192 simulated diagnostic SNPs distributed accordingly, with varying marker numbers and positions for each chromosome $(n=X=27)$, were generated in a map with total length of 2,811.2 cM. Populations with different segregation structures (F2, BC1-6) and expected frequencies of diagnostic markers (Table 1), each containing 10,000 individuals, were subsequently generated. A subset of 486 diagnostic markers with a minimal distance of $5 \mathrm{cM}$ were selected and subsequently used for hybrid identification in all simulated populations.

The most extreme scenario expected in current commercial Tambaqui broodstock populations, represented by a sixth backcross (BC6) generation $(\mathrm{F}(12)=p=0.015625)$, was used to determine minimal sampling size required for accurately estimating error rates of hybrid identification in each of the tested populations. A total of 100 random samplings, with replacement, of each considered size (10-100 individuals, in increments of $10 ; 100-500$, in increments of 50; $500-1,000$, in increments of $100 ; 1500 ; 2,000-10,000$ in increments of 1,000$)$ were performed, and rates of correct hybrid identification and respective standard errors determined, based on a total of 486 genotyped diagnostic markers selected. The estimated point where the curve reached an optimal plateau, considering a linear-plateau segmented regression model, was used to determine the minimal number of individuals to be sampled from each population type for proper comparisons with calculated error rates for hybrid identification (Figure 1).

Genotypes from 486 SNP markers from 100 repeated samplings of 300 individuals from each simulated hybrid population (F2, BC1-6) were used for identification of hybrids and computation of error rates. Following the initial sampling and computations, 27 markers were randomly removed (one marker per chromosome), individuals were randomly sampled again and error rates computed. This procedure was repeated for 13 additional rounds, followed by 26 rounds where a single maker was removed per round. Therefore, the total number of markers used for computation of error rates varied from 486 and 1 in each simulated popu-

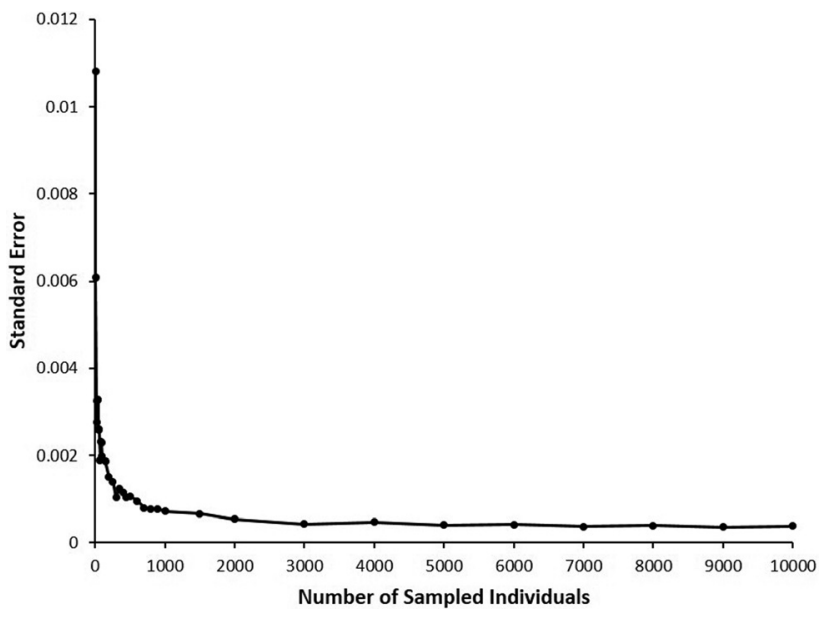

Figure 1 - Standard errors for correct identification of hybrid individuals in a simulated population after six generations of backcrossing (BC6) with a target species, obtained using different numbers of sampled individuals from a simulated population with 10,000 individuals.

lation type. Non-linear equations were derived for BC5 and BC6 observed error rate curves.

\section{Results}

\section{Estimated minimal number of markers required for hybrid detection based on power analysis}

The critical value for $p$ used for calculating expected numbers of independent bi-allelic markers required to identify hybrids in different intercross and backcross populations of a target species with a genome size of $1.2 \mathrm{Gbp}$ was determined to be 7.62939E-10 (Figure 1). An extreme situation was considered where every nucleotide could be considered a potential diagnostic marker, and therefore 30 generations of backcrossing an F1 hybrid would be required for the expected number of base-pairs from a non-target species to be $<1 b$. Figure 2 shows expected numbers of base-pairs from a

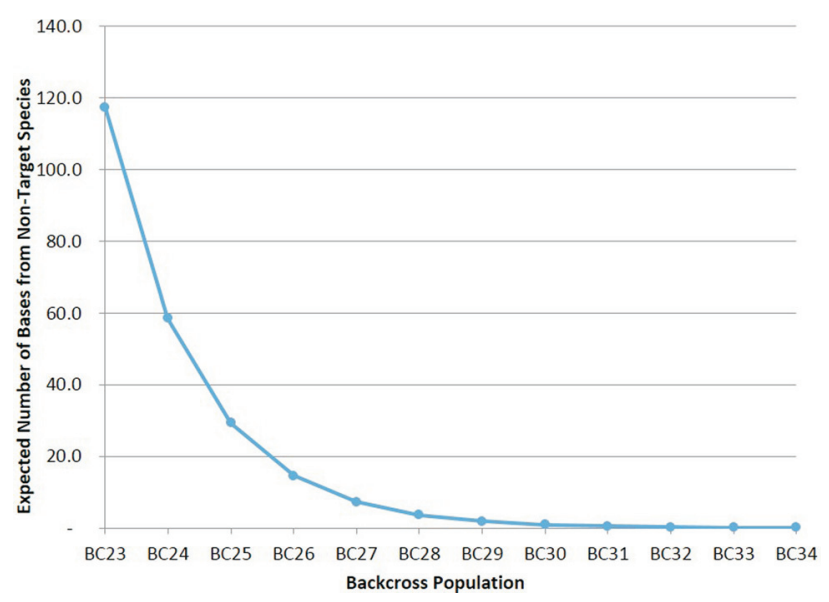

Figure 2 - Expected number of base-pairs (E) from a non-target species, considering number of backcross generation with a target species with a genome of $1.2 \mathrm{Gbp}$. 
non-target species across backcross generations 1 to 34 for a species with a genome with $1.2 \mathrm{Gbp}$. The relationship of genome size with number of base-pairs from a non-target species expected considering number of backcross generation, is shown on Figure 3.

Table 2 shows the estimated numbers of diagnostic independent bi-allelic markers required to identify hybrids in different intercross and backcross populations between a target and a non-target species based on power analysis, considering $p=7.62939 \mathrm{E}-10$, with $\operatorname{Power}(1-\beta) \cong 99 \%$, as in equation 1. Estimated numbers of required markers ranged from 5 to $191(\alpha=.05)$, and from 7 to 293 ( $\alpha=.01)$, considering populations $\mathrm{BC} 1$ to $\mathrm{BC} 6$, respectively (Figure 4).

\section{Simulations}

Figure 1 shows the relationship between number of sampled individuals and standard error rates for hybrid identification in the most extreme intercrossed population con-

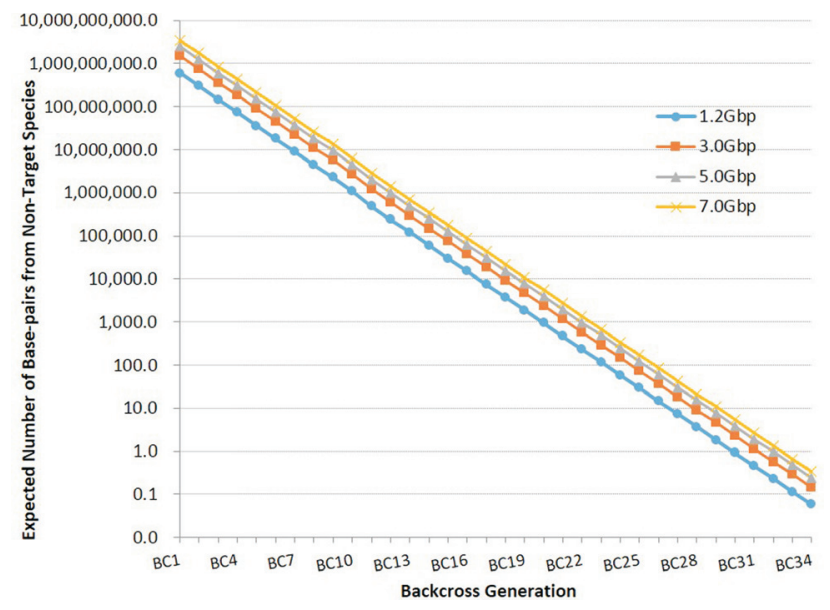

Figure 3 - Expected number of base-pairs from a non-target species considering number of backcross generation with a target species with genome size varying from 1.2 to $7.0 \mathrm{Gbp}$.

Table 2 - - Estimated numbers of bi-allelic markers required to identify hybrid individuals in different intercross and backcross populations between a target and a non-target species based on power analysis, considering $p=7.62939 \mathrm{E}-10$ and Power $(1-\beta) \cong 99 \%$, and observed on simulated populations, respectively.

\begin{tabular}{lcccc}
\hline $\begin{array}{l}\text { Population } \\
\text { Type }\end{array}$ & $\begin{array}{c}\text { Estimated numbers based on } \\
\text { power analysis }\end{array}$ & \multicolumn{2}{c}{$\begin{array}{c}\text { Observed numbers based } \\
\text { on simulated populations }\end{array}$} \\
\hline & $\alpha=.05$ & $\alpha=.01$ & $\begin{array}{c}\text { Error Rate } \\
\leq 5 \%\end{array}$ & $\begin{array}{c}\text { Error Rate } \\
\leq 1 \%\end{array}$ \\
\hline F2 & 3 & 4 & 3 & 4 \\
BC1 & 5 & 7 & 5 & 7 \\
BC2 & 11 & 17 & 11 & 17 \\
BC3 & 23 & 35 & 23 & 54 \\
BC4 & 47 & 72 & 54 & 135 \\
BC5 & 95 & 145 & 189 & $1,616^{*}$ \\
BC6 & 191 & 293 & $1,131^{*}$ & $8,065^{*}$ \\
\hline
\end{tabular}

*Calculated using non-linear equations derived from observed error rate curves in simulated populations (Figure 5)

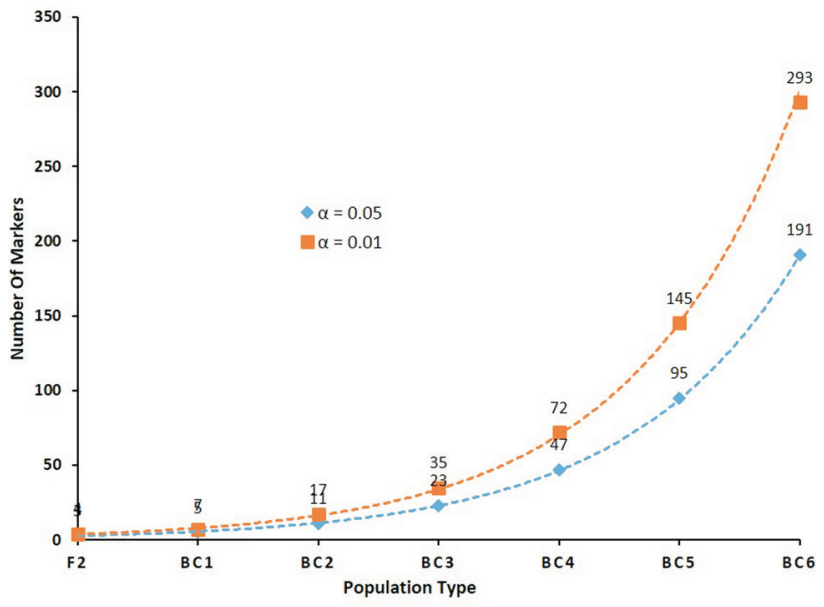

Figure 4 - Estimated numbers of independent bi-allelic markers expected for correctly identifying hybrid individuals in different intercross and backcross populations between a target and a non-target species based on power analysis, considering $p=7.62939 \mathrm{E}-10$, Power $(1-\beta) \cong 99 \%$, and $\alpha$ $=.05$ or .01 .

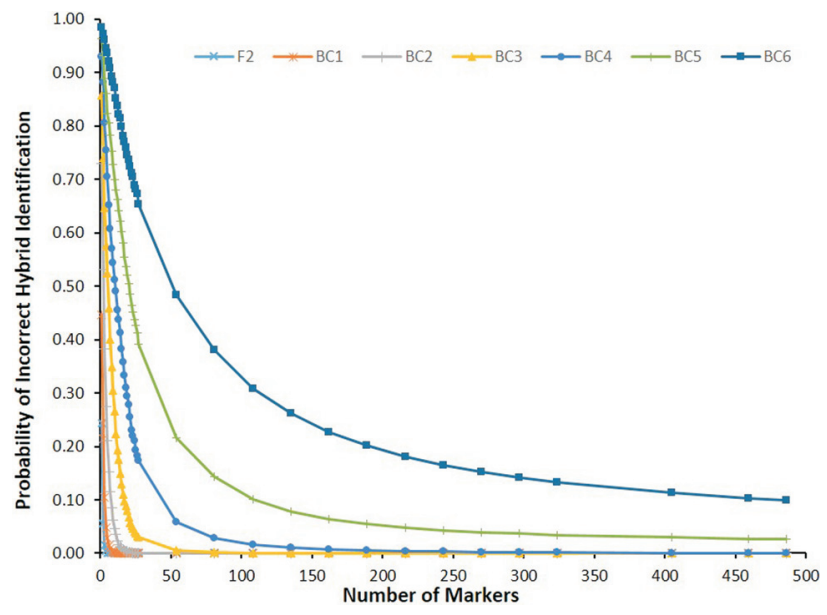

Figure 5 - Probability of incorrect identification of hybrid individuals observed in different types of simulated inter and backcross populations, considering increasing numbers of diagnostic markers.

sidered (BC6). The estimated point where the curve reaches an optimal plateau was $258.716 \pm 41.983$ individuals, considering a linear-plateau segmented regression model, which represents the minimal number of individuals that should be sampled for accurate calculation of error rates for hybrid identification. Subsequently, 100 independent samples of 300 simulated individuals were checked for correct hybrid identification, for accurate calculation of error rates in all remaining population types and numbers of diagnostic markers considered.

Observed error rates for correct hybrid identification in all simulated types of inter and backcross populations using from 1 to 486 markers are shown in Table S1. Figure 5 shows observed error rate curves observed for each simulated population. Observed numbers of markers required to identify hybrids in simulated populations (Table 2) were compared with expected numbers (Figure 6). Non-linear 


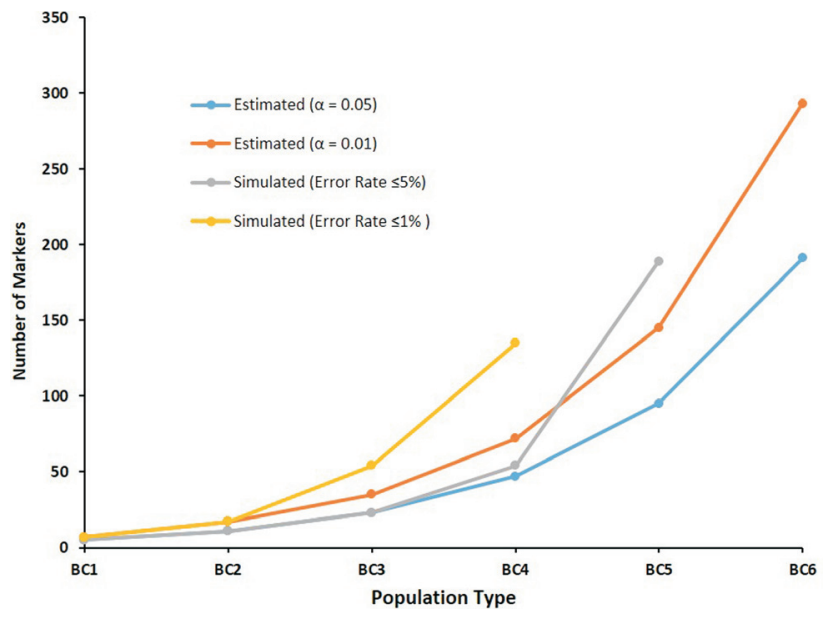

Figure 6 - Comparisons of estimated numbers of independent bi-allelic markers required to identify hybrid individuals in different intercross and backcross populations based on power analysis and observed error rates in simulated populations.

equations derived for $\mathrm{BC} 5(Y \sim 6.36973+$ $\left.4.51791 X_{-1.27593}, \mathrm{R}^{2}=99.5 \%\right)$ and BC6 $(Y \sim-26.0083+$ $30.96739 X^{1.20855}, R^{2}=99.9 \%$ ), were used to calculate numbers of markers $(Y)$ required for accurate hybrid identification with error rate $X$ in BC5 and BC6 (Table 2). Critical command lines used in simulations with $\mathrm{R} / \mathrm{qtl}$ are provided in File $\mathrm{S} 1$.

\section{Discussion}

Proper validation of molecular diagnostic tools for identification of cross-species hybrid individuals requires adequate statistical tests to determine expected error rates and confidence levels associated with the number and type of markers used, considering respective analytical limits associated with admixture and introgression levels in target populations, in addition to other innate error sources (i.e. genotyping errors). Numbers of required markers for correct hybrid identification estimated and simulated herein show that a minimum of four or seven independently-segregating nuclear bi-allelic markers with species-specific alleles are required for accurate individual hybrid identification in F2 and $\mathrm{BC} 1$ populations, respectively, considering false positive $(\alpha)$ error rates $<1 \%$. Conversely, an estimated minimum of up to 95 and 145 markers are required for accurate hybrid identification, considering $\alpha$ of $5 \%$ and $1 \%$, respectively, when introgression levels considered are down to $1.56 \%$, which is expected in a BC5.

Proposed tests for identification of Siluriforme hybrids (Pseudoplatystoma corruscans and P. reticulatum) have been based on a single nuclear and a single mitochondrial marker with species-specific alleles (Prado et al., 2011), or on eight microsatellite markers with differing allele frequencies with no exclusive species-specific alleles (Carvalho et al., 2013). Similarly, a molecular test for identification of Serrasalmid hybrids (Colossoma macropomum, Piaractus mesopotamicus and P. brachypomus) was proposed based on two nuclear markers and two mitochondrial markers (Hashimoto et al., 2011). In both groups of species, mitochondrial markers are haploid and inherited maternally. These examples include species frequently hybridized in commercial aquaculture operations for production of fry destined to grow out and human consumption, which generate fully fertile hybrids that can be readily intercrossed or backcrossed to pure parental species. Obtained estimates and observations in simulated populations herein show that significantly higher numbers of independently segregating markers with species-specific alleles are required for accurate identification of advanced-cross hybrids and proper certification and management of pure species broodstocks, than may be currently under use considering existing proposed tests. Diagnostic tests based on 2-3 markers with speciesspecific alleles may be appropriate for identification of F1 crossbreds but, considering observed results, are far from having sufficient analytical power for identifying introgressions which may eventually have negative impacts on genetic improvement programs and conservation of natural populations.

Methods using allozyme and microsatellite data to identify hybrids and introgressions based on allele frequency differences between populations have been applied to identify F1, F2 and backcross 1 (BC1) hybrids in simulated data from wild brown trout (Salmo truta) populations from areas stocked with hatchery fish (Sanz et al., 2009). In addition, studies based on population allele frequency differences using SNP data derived from restriction-associated DNA (RAD-Seq) sequencing (Baird et al., 2008) have identified hybrids between two closely related sole species (Souissi et al., 2018), and European (Anguilla anguilla) and American (A. rostrata) eels (Pujolar et al., 2014). Even though the cited methods clearly identified the occurrence of hybridization events, hybrid individuals beyond the $\mathrm{F} 2$ or $\mathrm{BC} 1$ levels could not be accurately identified, and therefore such methods could not be used for routine diagnostics and certification of individual fish in situations where broodstock have been kept captive for several generations.

Listed formulae and performed simulations considered a number of critical assumptions, including uniform distribution and independent segregation of markers, marker neutrality, and Hardy-Weinberg equilibrium in advanced intercrossed and backcrossed hybrid populations, as have other similar studies performed to estimate exclusion probabilities in random mating and similar types of structured populations (Jamieson and Taylor, 1997; Saunders et al., 2007; Baruch and Weller, 2008; Carvalho et al., 2013). Considering the proposed framework is based on the use of extensively validated markers, shown to have species-specific alleles, Type I errors error rates $(\alpha)$ associated with not identifying a true hybrid are expected to result from genotyping errors, de novo mutations, and other issues addressed subsequently, which may result in a lack of assayed markers in genome regions introgressed from non-target species.

Deviations between estimated and observed numbers of markers required for correct identification of hybrids in 
simulated populations were observed in advanced backcross populations (Table 2, Figure 6). Calculations of expected numbers of markers considered complete linkage independence between diagnostic markers. However, considering the number of effective independent markers is finite, observed differences between estimated and observed numbers of required markers can be attributed to the lack of independent assortment between markers with distances $<50 \mathrm{cM}$. In the particular simulated species $n=X=27$, and deviations between calculated and observed numbers of makers required for accurate hybrid identification were observed to emerge when $m>27$. Moreover, resulting residual genome fragments from a non-target species in advanced backcrosses may be distributed in increasingly larger numbers of smaller chromosome fragments, with diminishing probabilities of presence of at least one diagnostic marker, as a consequence of subsequent recombination events across generations, and may be affected by species-specific recombination rates. Consequently, deviations between calculated and observed numbers of markers required for accurate hybrid identification may be even higher than expected because of lack of independence, which may explain the observed 27-fold difference between expected and observed numbers of markers required for accurate hybrid identification in a BC6 $(\alpha<.01)$. Therefore, final determination of minimal numbers of diagnostic markers required for species-purity certification should consider chromosome number, average recombination rate and supposed number of generations when earliest introgressions may have occurred in target populations.

Calculations of test power require the use of critical non-zero values for $p$, as $p$ approaches zero, for solving equation (1). Instead of using a random $\sim 0$ value for $p$, an actual value was calculated considering the expected number of generations of backcrossing an F1 hybrid that would be required to remove every nucleotide from a non-target species from a genome with $1.2 \mathrm{Gbp}$ (30 generations). Critical non-zero values for $p$ calculated for genomes up to 7.0Gbp (Figure 2) were similarly observed after 33 generations of backcrossing, therefore indicating that genome size will have little effect on numbers of markers required for accurate hybrid identification. Calculations of marker numbers with larger $p$ values $(>.0001)$ were performed (data not shown) but did not affect observed results.

A segmented regression model was applied to determine minimal sampling size required for accurate estimation of error rates for hybrid identification in different types of simulated populations (Figure 3). Based on this finding, multiple samplings of 300 individuals from each tested population were therefore considered to be sufficient for accurate calculation of error rates in all studied populations. Simulations were limited to a maximum of 486 markers (18 per chromosome) as initial calculations indicated $<300$ markers would provide sufficient statistical power for accurate hybrid identification even in BC6 populations $(\alpha=.01)$. Unwanted introgressions in captive populations are likely to follow complex admixture patterns, similarly to natural pop- ulations. However, allele frequencies in late-generation hybrid populations resulting from these processes are likely to resemble those expected in advanced backcrossed populations. Considering generation intervals in these particular species are 3-4 years, and that most of current commercial broodstocks are descendants of animals captured in nature since year 2000 (Hashimoto et al., 2012), the most extreme possible case currently expected of an advanced-cross hybrid would be a BC5, falling within the limit of the most advanced backcross population (BC6) used for all calculations and simulations.

Estimated and observed numbers of diagnostic markers required for accurate hybrid identification up to four generations of backcrossing (BC4) fall well within practical operational limits of most commercial platforms currently available for genotyping low density SNP marker panels. Therefore, cost-effective assay panels could be developed to provide practical tools for accurate species-purity certification of individuals and management of commercial broodstocks, and monitoring of wild populations. In addition, proper numbers of diagnostic markers may be included within medium (tens of thousands) and high-density (hundreds of thousands) marker panels already available or under development for applications such as genome-wide genetic evaluations and selection (Tsai et al., 2015). Additional strategies using data from multiple adjacent SNPs generated with medium and high-density panels have also been developed to identify population-specific haplotypes (Halbert et al., 2005; Simcic et al., 2015), which are particularly useful when exclusive SNP variants are not available, and that can in turn be used to identify admixed individuals and eventually reconstitute original genetic backgrounds, using specifically designed breeding strategies. Reported genotyping errors for SNP markers vary according to platform and can be as low as $0.1 \%$ (Saunders et al., 2007). Considering that certifying species purity at the $\mathrm{BC} 4$ level will require $>100$ genotyped markers, requiring at least two conflicts to classify an individual as a hybrid may be justified, even if genotyping error rates are as low as cited by technology providers.

The methodology described herein may also be applied to determine minimal numbers of diagnostic markers required for identification of crossbreds derived from intercrossing different breeds/populations within a species. Tools for breed allocation for establishment of genetic resource conservation populations (Negrini et al., 2007) and certification of animal-derived food products (Sasazaki et al., 2007) have been developed, considering minimal to zero levels of inter or backcrossing of subjects. As shown, the high correlation observed between admixture level and expected numbers of diagnostic markers required to identify hybrids should be considered in cases where low error rates can be accepted in identifying subjects with low levels of introgressions from other populations/breeds. 


\section{Conclusions}

The obtained results established a statistical ground work for performing molecular diagnostic tests to identify individuals generated from undesired cross-species hybridizations with respective confidence levels, in different types of inter and backcross populations. Estimated and observed numbers of diagnostic markers required for proper individual hybrid identification up to four generations of backcrossing fall well within practical operational limits of most commercial platforms currently available for genotyping low-density SNP marker panels. Therefore, cost-effective assay panels could be developed to provide practical tools for accurate routine species-purity certification and management of commercial broodstocks and monitoring of wild populations.

\section{Acknowledgments}

Funding was provided by Embrapa, from grant 01.11.07.002.07.00. ARC is a CNPq fellow.

\section{Conflicts of Interest}

The authors declare that there is no conflict of interest that could be perceived as prejudicial to the impartiality of the reported research.

\section{Author Contributions}

JPS and ARC conceived the study, conducted the experiments, analyzed the data and wrote the manuscript. Both authors read and approved the final version.

\section{References}

Adams C (2007) Land transport in Roman Egypt: a study of economics and administration in a Roman Province. Oxford University Press, 346 p.

Baird NA, Etter PD, Atwood TS, Currey MC, Shiver AL, Lewis ZA, Selker EU, Cresko WA and Johnson EA (2008) Rapid SNP discovery and genetic mapping using sequenced RAD markers. PloS One 3:e3376.

Bartley DM, Rana K and Immink AJ (2001) The use of interspecific hybrids in aquaculture and fisheries. Rev Fish Biol Fish 10:325-337.

Baruch E and Weller JI (2008) Estimation of the number of SNP genetic markers required for parentage verification. Anim Genet 39:474-479.

Broman KW, Wu H, Sen S and Churchill GA (2003) R/qtl: QTL mapping in experimental crosses. Bioinformatics 19:889-890.

Carvalho DC, Seerig AS, Brasil BSAF, Crepaldi DV and Oliveria DAA (2013) Molecular identification of the hybrid between the catfish species Pseudoplatystoma corruscans and Pseudoplatystoma reticulatum using a set of eight microsatellite markers. J Fish Biol 83:671-676.

Halbert ND, Ward TJ, Schnabel RD, Taylor JF and Derr JN (2005) Conservation genomics: disequilibrium mapping of domestic cattle chromosomal segments in North American bison populations. Mol Ecol 14:2343-2362.
Hashimoto DT, Mendonca FF, Senhorini JA, Bortolozzi J, Oliveira C, Foresti F and Porto-Foresti F (2010) Identification of hybrids between Neotropical fish Leporinus macrocephalus and Leporinus elongatus by PCR-RFLP and multiplex-PCR: tools for genetic monitoring in aquaculture. Aquaculture 298:346-349.

Hashimoto DT, Mendonca FF, Senhorini JA, Oliveira C, Foresti F and Porto-Foresti F (2011) Molecular diagnostic methods for identifying Serrasalmid fish (Pacu, Pirapitinga, and Tambaqui) and their hybrids in the Brazilian aquaculture industry. Aquaculture 321: 49-53.

Hashimoto DT, Prado FD, Senhorini JA, Foresti F and Porto-Foresti F (2012) Detection of post-F1 fish hybrids in brood stock using molecular markers: approaches for genetic management in aquaculture. Aquac Res 1:1-9.

Heaton MP, Keen JE, ClawsLon ML, Harhay GP, Bauer N, Shultz C, Green BT, Durso L, Chitko-McKown CG and Laegreid WW (2005) Use of bovine single nucleotide polymorphism markers to verify sample tracking in beef processing. J Am Vet Med Assoc 226:1311-1314.

Jamieson A and Taylor SS (1997) Comparisons of three probability formulae for parentage exclusion. Anim Genet 28:397-400.

Mair GC (2007) Genetics and breeding in seed supply for inland aquaculture. In: Bondad-Reantaso MG (ed) Assessment of freshwater fish seed resources for sustainable aquaculturepp. FAO Fisheries Technical Paper, Rome.

Negrini R, Milanesi E, Colli L, Pellecchia M, Nicoloso L, Crepaldi P, Lenstra JA and Ajmone-Marsan P (2007) Breed assignment of Italian cattle using biallelic AFLP markers. Anim Genet 38:147-153.

Nunes JR, Liu S, Pértille F, Perazza CA, Villela PM, Almeida-Val VM, Hilsdorf AW, Liu Z and Coutinho LL (2017) Large-scale SNP discovery and construction of a high-density genetic map of Colossoma macropomum through genotyping-by-sequencing. Sci Rep 7:46112.

Pritchard JK, Stephens M and Donnelly P (2000) Inference of population structure using multilocus genotype data. Genetics 155:945-959.

Prado FD, Hashimoto DT, Mendonça FF, Senhorini JA, Foresti F and Porto-Foresti F (2011) Molecular identification of hybrids between Neotropical catfish species Pseudoplatystoma corruscans and Pseudoplatystoma reticulatum. Aquac Res 42:1890-1894.

Pujolar JM, Jacobsen MW, Als TD, Frydenberg J, Munch K, Jónsson B, Jian JB, Cheng L, Maes GE, Bernatchez L et al. (2014) Genome-wide single-generation signatures of local selection in the panmictic European eel. Mol Ecol 23:2514-2528.

Sanz N, Araguas RM, Fernandez R, Vera M and Garcia-Marin JL (2009) Efficiency of markers and methods for detecting hybrids and introgression in stocked populations. Conserv Genetic 10:225-236.

Sasazaki S, Mutoh H, Tsurifune K and Mannen H (2007) Development of DNA markers for discrimination between domestic and imported beef. Meat Sci 77:161-166.

Saunders IW, Brohede J and Hannan GN (2007) Estimating genotyping error rates from Mendelian errors in SNP array genotypes and their impact on inference. Genomics 90:291-296.

Simcic M, Smetko A, Sölkner J, Seichter D, Gorjanc G, Kompan D and Medugorac I (2015) Recovery of native genetic background in admixed populations using haplotypes, phenotypes, and pedigree information - using Cika cattle as a case breed. PLoS One. 10:e0123253. 
Souissi A, Bonhomme F, Manchado M, Bahri-Sfar L and Gagnaire PA (2018) Genomic and geographic footprints of differential introgression between two divergent fish species (Solea $\mathrm{spp}$.). Heredity 121:579-593.

Tsai HY, Hamilton A, Tinch AE, Guy DR, Gharbi K, Stear MJ, Matika O, Bishop SC and Houston RD (2015) Genome wide association and genomic prediction for growth traits in juvenile farmed Atlantic salmon using a high density SNP array. BMC Genomics 16:969.

Vignal A, Milan D, SanCristobal M and Eggen A (2002) A review on SNP and other types of molecular markers and their use in animal genetics. Genet Sel Evol 34:275-305.

\section{Supplementary material}

The following online material is available for this article: Table S1 - Probabilities of error in identifying a true individual hybrid with different numbers of di-allelic markers with species-specific alleles observed in different types of simulated populations.

File S1 - Critical parameters used in simulations with R/qtl.

Associate Editor: Vera M. Fonseca de Almeida e Val

License information: This is an open-access article distributed under the terms of the Creative Commons Attribution License (type CC-BY), which permits unrestricted use, distribution and reproduction in any medium, provided the original article is properly cited. 